SUBJECT AREAS:

SOLAR ENERGY AND

PHOTOVOLTAIC

TECHNOLOGY

MICRORESONATORS

Received

7 February 2014

Accepted

23 April 2014

Published

14 May 2014

Correspondence and requests for materials should be addressed to J.M. (Jordi.martorell@ icfo.es) \section{Gallery Modes of a fiber array thin film solar cell for fixed partial Sun tracking}

\author{
Marina Mariano' ', Francisco J. Rodríguez' ', Pablo Romero-Gomez' , Gregory Kozyreff² \& Jordi Martorell',3
}

\begin{abstract}
'Organic Nanostructured Photovoltaics, ICFO - The Institute of Photonic Sciences. Mediterranean Technology Park, 08860 Castelldefels (Barcelona, Spain), ${ }^{2}$ Département de physique, ULB - Université Libre de Bruxelles. Campus de la Plaine, CP 231 1050, Bruxelles (Belgium), ${ }^{3}$ Departament de Física i Enginyeria Nuclear, Universitat Politècnica de Catalunya. 08222, Terrassa, (Spain).
\end{abstract}

We propose the use of whispering gallery mode coupling in a novel configuration based on implementing a thin film cell on the backside of an array of parallel fibers. We performed numerical calculations using the parameters of a thin film organic cell which demonstrate that light coupling becomes more effective as the angle for the incident light relative to the fiber array normal increases up to an optimal angle close to $55 \mathrm{deg}$. At this angle the power conversion efficiency of the fiber array solar cell we propose becomes $30 \%$ times larger than the one from an equivalent planar cell configuration. We demonstrate that the micro fiber array solar cell we propose may perform an effective partial tracking of the sun movement for over 100 degrees without any mechanical help. In addition, in the event that such fiber array cell would be installed with the adequate orientation on a vertical façade, an optimal photon-to-charge conversion would be reached for sunlight incident at $55 \mathrm{deg}$ with respect to the horizon line, very close to the yearly average position for the sun at Latitude of $40 \mathrm{deg}$.

$\mathrm{n}$ thin film devices that use light as the energy source, it is of the utmost importance to implement an adequate material or structure configuration to obtain an optimal light absorption. In organic solar cells where the thickness of the active material is, typically, about one hundred nanometers, several approaches have been implemented, recently, to increase the effective light path ${ }^{1-4}$. Using the light scattered by plasmonic nanoparticles to achieve a $20 \%$ enhancement in the light absorption, single junction organic cells with a power conversion efficiency above $8 \%$ were demonstrated ${ }^{5}$. A disordered multilayer architecture with similar properties to onedimensional photonic crystals has been used to enhance near infrared and near UV light absorption in semitransparent organic cells which exhibited a power conversion efficiency above $5 \%{ }^{6}$. Structured substrates forming an array of microprisms ${ }^{7}$ or a curved surface such as the one obtained when spheres are laid over a planar device have also been considered to enhance light harvesting. A configuration where spheres stood in the proximity to a high-index photovoltaic absorber layer from a thin film cell was proposed to couple light into such high-index material and to obtain an enhanced light absorption. The authors claimed that the more effective coupling originated from whispering gallery modes (WGM) inside the spheres ${ }^{8}$. An alternative approach to improve absorption considered forming whispering-gallery resonant modes inside a low quality-factor spherical nanoshell structure that facilitated the coupling of light into the resonant modes and substantially enhanced the light path in the active material ${ }^{9}$. Coupling into high quality factor WGM has been extensively considered and has produced very encouraging results for the generation of laser light ${ }^{10-14}$, nonlinear light ${ }^{15-21}$ or sensing ${ }^{22-26}$.

In the current work, we propose the use of WG coupling but in a drastically different configuration based on implementing an organic cell on the back side of an array of parallel fused silica fibers. In such an array, light can be coupled into low quality factor WG resonant modes of the fibers. Such coupling becomes more effective as the angle for the incident light relative to the normal of the fiber array increases up to $55 \mathrm{deg}$. In any case, as we will show below, light absorption at the active layer is at all angles larger for the fiber array configuration when compared to the standard planar configuration. In addition, the specific angular dependence of the light absorption in the configuration under study may provide an optimal solution to mimic sun-tracking systems or to enhance light harvesting for vertical PV installations. 

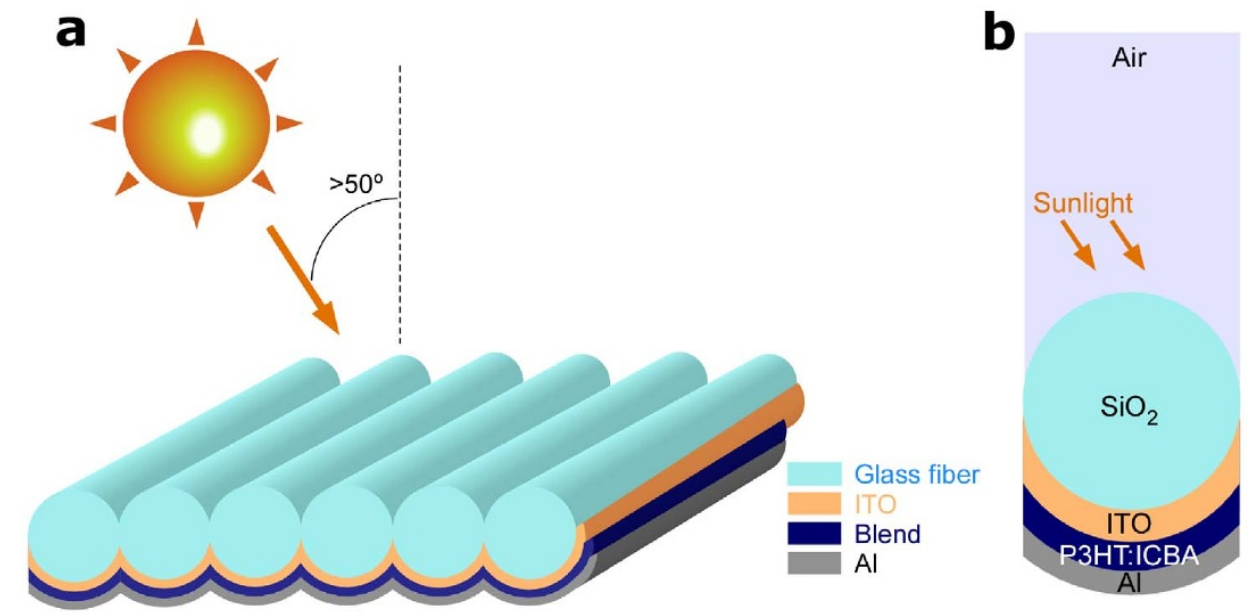

Figure $1 \mid$ Schematic view of the FAOC. a)The fiber array is the substrate where the solar cell device is deposited. The performance of the solar device is studied as a function of the angle of incidence (theta) of the Sunlight relative to the normal to the fiber array. b) Enlarged view of the cross section of one silica fiber from the array shown in a).

\section{Results}

A schematic view of the solar cell device considered in here can be seen in Figure 1a. The device may consist of a number of fibers stuck together forming an array of parallel fibers that serve as the substrate for an organic solar cell device. The different layers of the cell architecture are deposited on the back side of the array relative to the incoming sunlight incident on the front side of the array. A detailed cross section of a single cell fiber from the array is shown in Figure 1b. For the numerical study we present below, we consider silica as the substrate material. The back side of this substrate is coated with a three layer organic cell composed of a layer of Indium Tin Oxide (ITO) as transparent electrode, an active layer made of a a

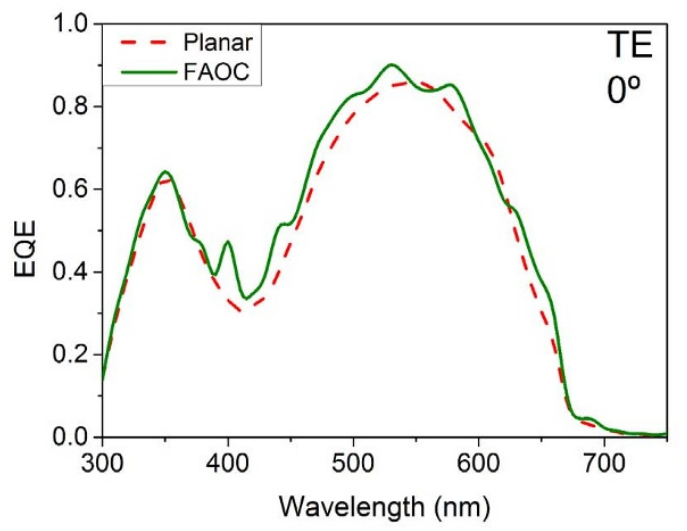

C

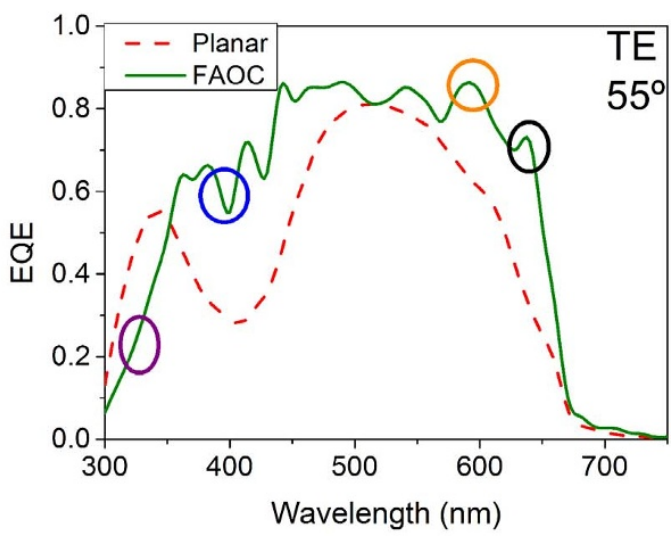

b

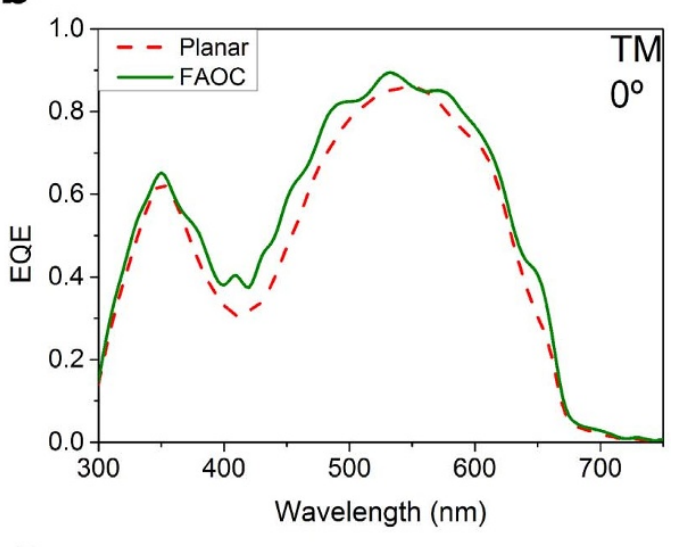

d

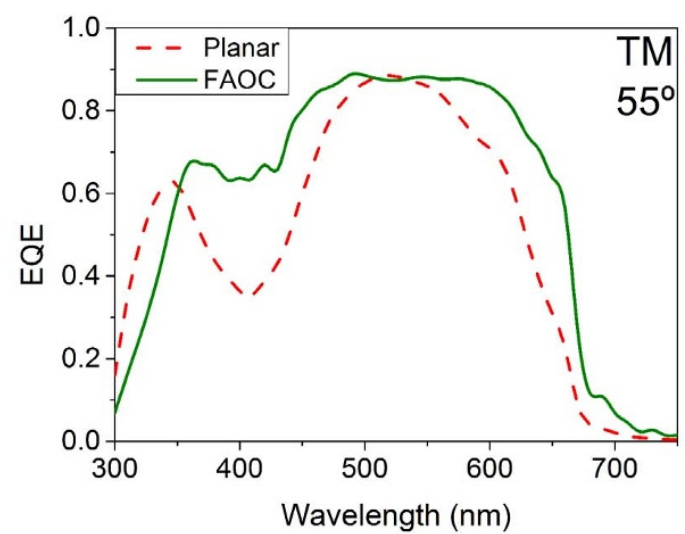

Figure $2 \mid$ External quantum efficiencies of a $1.5 \mu \mathrm{m}$ diameter fiber FAOC (solid green) and an equivalent planar cell (dashed red). The simulated EQEs are shown at normal incidence ( $0 \mathrm{deg}$ ) for a) TE and b) TM polarizations and at $55 \mathrm{deg}$ for c) TE and d) TM polarizations. Note that at 55 deg incidence the increase in EQE for the fiber array relative to the planar cell is apparent at almost all wavelengths. The TE field intensity distributions at $320 \mathrm{~nm}$ (violet circle), $400 \mathrm{~nm}$ (blue circle), $600 \mathrm{~nm}$ (orange circle), and $640 \mathrm{~nm}$ (black circle) are shown in Figure 3. 

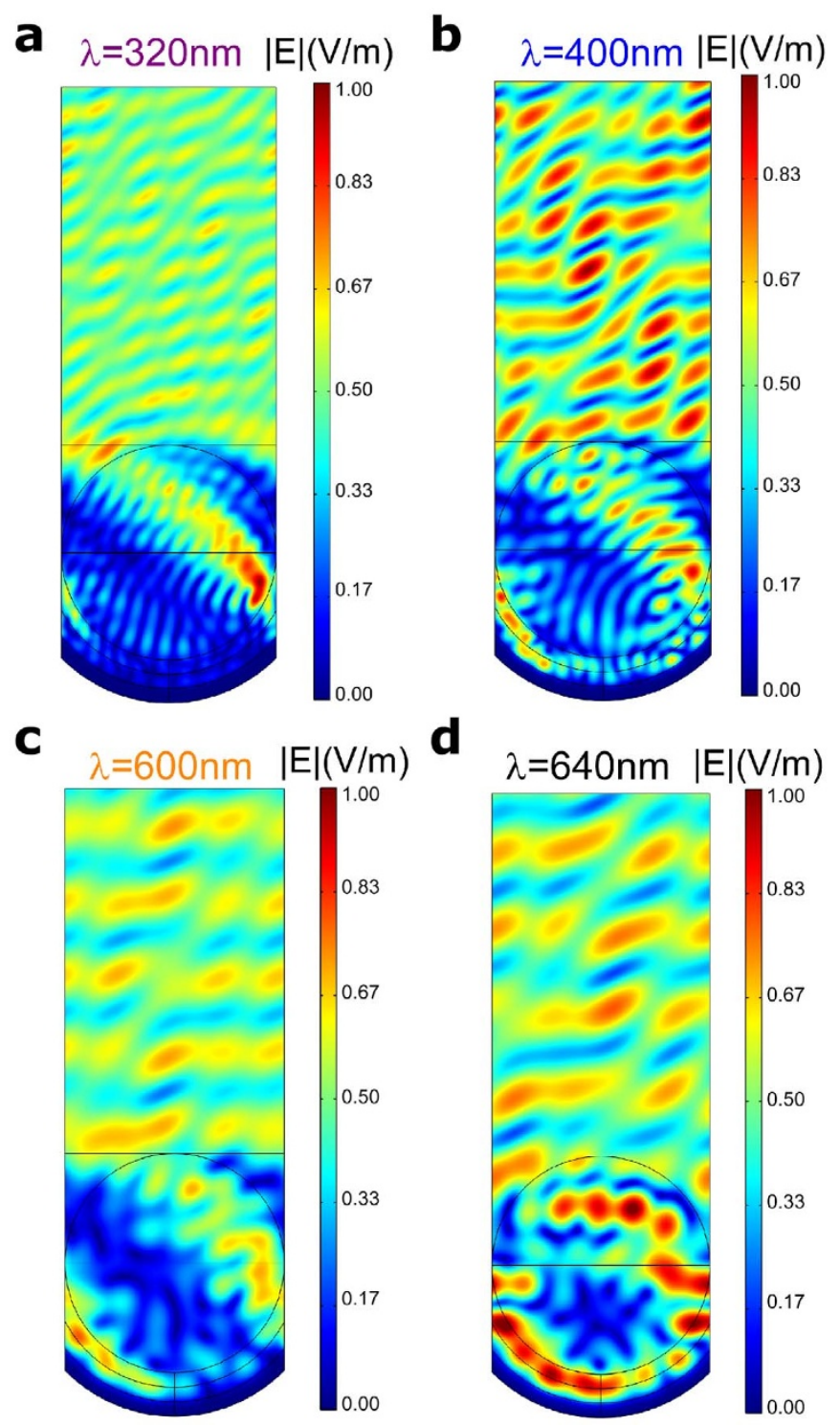

Figure $3 \mid$ Cross section of the spatial distribution TE field norm. Fiber diameter of the FAOC is $1.5 \mu \mathrm{m}$ and light is incident at $55 \mathrm{deg}$, and the wavelength tuned at a) $320 \mathrm{~nm}$, b) $400 \mathrm{~nm}$, c) $600 \mathrm{~nm}$ and d) $640 \mathrm{~nm}$. All figures are normalized to $1 \mathrm{~V} / \mathrm{m}$ and the same color coding is used for all of them.

Poly(3-hexylthiophene-2,5-diyl) blended with Indene-C60 Bisadduct (P3HT:ICBA), which extinction coefficient is shown in Figure $\mathrm{SI} 2 \mathrm{~b}$, and an Aluminum (Al) metallic contact. Extremely thin layers that must be included in an experimental realization of the majority of organic cells, as noted in the supplementary information file, were omitted in our numerical calculations given their negligible effect in the re-distribution of the optical field intensity.

The convolution of the P3HT:ICBA extinction coefficient with the sun photon flux leaves a wavelength window for PV operation ranging from $300 \mathrm{~nm}$ to $750 \mathrm{~nm}$. At each wavelength, the total power dissipation over the active layer area is integrated to extract the photon absorption in this layer or, equivalently, the External Quantum Efficiency (EQE). Provided that the scope of the work is to compare the performance of such fiber array organic cell (FAOC) relative to the planar configuration, the wavelength independent factor to account for losses in the collection of charges relative to the absorbed photons, is taken to be equal to unity. To validate the reliability of the numerical model used, the experimentally measured EQE from a fabricated planar cell is compared to the numerically calculated one in figures SI1 from the supplementary information file. The predicted EQEs at normal incidence $(0 \mathrm{deg})$ for the FAOC and planar cells at, both, TE and TM polarizations are shown in Figure $2 \mathrm{a}$ and $2 \mathrm{~b}$, respectively. Although the performance of the FAOC is slightly better than the planar one, no significantly different features are observed when comparing both EQEs. This is not the case, when the light is incident at $55 \mathrm{deg}$. In that event, the EQE for the FAOC, shown for the TE and TM polarizations in Figures $2 \mathrm{c}$ and $2 \mathrm{~d}$, respectively, is largely improved at almost the entire wavelength range. In the wavelength range spanning from 430 to $600 \mathrm{~nm}$, the EQE is almost saturated to its maximum possible value. The EQE of the planar cell at $400 \mathrm{~nm}$ and surrounding wavelengths exhibits very low values reaching a local minimum of 0.25 for the TE polarization and 0.35 for the TM polarization due to the combined effect of a destructive interference and a local minimum of the P3HT:ICBA extinction coefficient (cf. Figure SI2b). On the contrary, in the same wavelength region the EQE for the FAOC is always higher than 0.5 for both polarizations.

To better understand what causes the increase in EQE at 55 deg incidence, we numerically computed the spatial electric field norm distribution at 320, 400, 600 and $640 \mathrm{~nm}$, shown in Figure 3 for the TE polarization. Such field distribution seems to indicate that for the last three cases photon trapping in the active layer occurs all along the half coated fiber perimeter. Incidence at 55 deg induces a partial excitation of a WGM, which leads to light trapping at the inner boundary of the spherical interface separating the transparent electrode and blend from the metal electrode. The excitation of WGMs is particularly apparent at $640 \mathrm{~nm}$ when the extinction coefficient is small. The TE field distribution, shown in Figure 3d, exhibits high intensity lobes in a large part of the half perimeter of the cylinder occupied by the ITO electrode and active layer. Therefore, we may attribute the 2.3 times enhancement observed in the EQE to an increased absorption path for the light within the active layer. At $320 \mathrm{~nm}$ coupling to the WGM is aborted by the higher extinction coefficient of ITO at that wavelength. Consequently, as seen in Figure 2, no gain in the EQE can be obtained at that wavelength region where absorption is dominated by the ITO extinction coefficient. We have also considered the case where the fiber array is finite and limited to a total number of five fibers surrounded by air. In that case the field distribution obtained was computed and, as shown in Figure SI4 in the supplementary information file, similar field distribution lobes as the ones from Figure 3 were observed. In accordance, the EQE of such finite FAOC is similar to the one from the periodic FAOC, as shown in Figure SI5 from the supplementary information file. These results confirm the validity of the periodic boundary conditions applied to model light harvesting in the FAOCs.

To estimate the short circuit current of the FAOC and compare it to the one from the standard planar, we computed the short circuit current $\left(\mathrm{J}_{\mathrm{sc}}\right)$ obtained at each angle of incidence, shown in Figure 4a. This current was calculated integrating the EQE multiplied by the solar photon flux and the electron charge $q$ over all wavelengths $\lambda$, as in the equation

$$
J_{s c}=\int E Q E(\lambda) \frac{I(\lambda) \lambda}{h c} q \mathrm{~d} \lambda
$$

where $I(\lambda)$ indicates the standard AM1.5G spectral irradiance of the Sun. At normal incidence, the $\mathrm{J}_{\mathrm{sc}}$ for the FAOC is $6 \%$ higher than the planar cell. When the angle of incidence increases the planar cell's $\mathrm{J}_{\mathrm{sc}}$ stays almost constant up to $40 \mathrm{deg}$, whereas for the FAOC one increases with angle as the light gets coupled more effectively into the WGM. The $\mathrm{J}_{\mathrm{sc}}$ exhibits a maximum at 55 deg being $30 \%$ larger than the planar. At higher angles, as expected, the $\mathrm{J}_{\mathrm{sc}}$ for, both, FAOC and planar cells decays rapidly but the one for the FAOC remains always larger.

As the angle of incidence increases, the electrical power generated for both cells would decrease by a cos(theta) factor accounting for the 

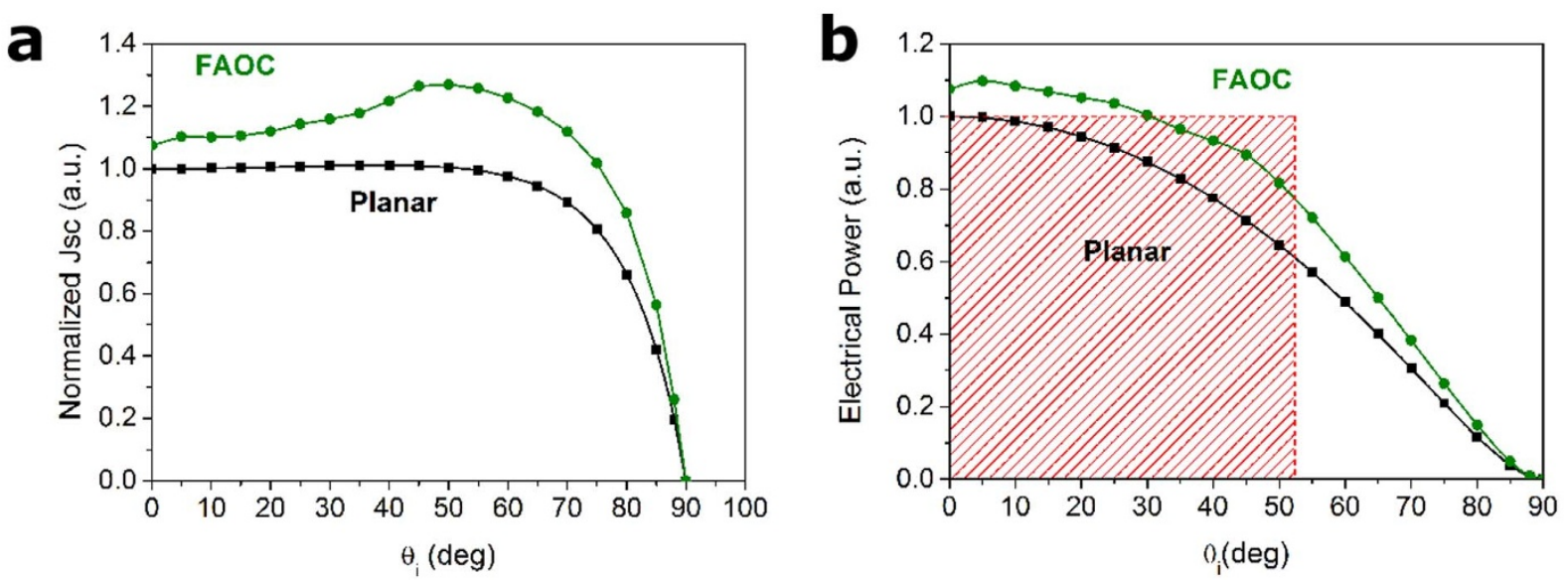

Figure $4 \mid$ Normalized electrical response of FAOC compared to a planar cell. a) Normalized $\mathrm{J}_{\mathrm{sc}}$ for the planar cell (black squares) and for a $1.5 \mu \mathrm{m}$ fiber diameter FAOC (green dots) as a function of the sunlight angle of incidence. b) Electrical power in arbitrary units as a function of the incidence angle provided by the planar device (black squares) and by the $1.5 \mu \mathrm{m}$ fiber diameter FAOC (green dots). The area of the red rectangle would be proportional to the energy collected by a planar cell tracking the movement of the sun up to $52 \mathrm{deg}$. Up to this angle the area under the FOAC curve is the same. Currents and powers are normalized to the response of the planar device at 0 deg. The black and green lines are only a guide for the eye.

projected area to a surface normal to the direction of the incident light. As shown in Figure 4b, this reduction in electrical power can be partially compensated by the FAOC due to a photocurrent generation that is maximized at $55 \mathrm{deg}$ rather than at normal incidence. For a solar cell placed in a horizontal plane, energy collected from sunrise to sunset would be proportional to two times the area underneath the curves shown in Figure $4 \mathrm{~b}$. This area is $17 \%$ times larger for the FAOC than for the planar case. As shown in Figure $4 \mathrm{~b}$ too, the energy collected by the FAOC from 0 to 52 deg would be equivalent to the energy collected by a planar cell maintained, at all times, perpendicular to the sun in that angular range.

At an incidence of 55 deg we studied the $\mathrm{J}_{\mathrm{sc}}$ dependence with the fiber diameter, keeping the other geometrical parameters fixed. At diameters below $400 \mathrm{~nm}$, when light confinement at the perimeter of the fiber close to the back metal contact is ineffective, the $\mathrm{J}_{\mathrm{sc}}$ for the FAOC is not better than for the planar configuration. As seen in Figure $5 \mathrm{a}$, this situation is reversed as the diameter is increased, and the $\mathrm{J}_{\mathrm{sc}}$ reaches a maximum at a fiber diameter of $1.75 \mu \mathrm{m}$. At such fiber dimensions the EQE, shown in Figure $5 b$, is very close to the optimal for a large wavelength interval spanning from 375 to

a

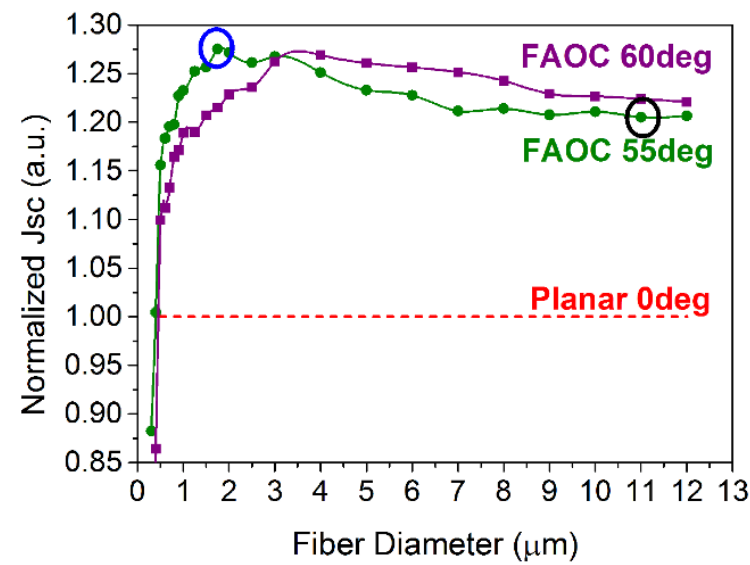

$650 \mathrm{~nm}$. This indicates an optimal coupling to the WGM for such entire wavelength range. As the fiber diameter is increased further, the $\mathrm{J}_{\mathrm{sc}}$ decreases while the EQE for the FAOC resembles more the one for the planar cell, as shown in Figure 5b. This indicates a less effective coupling to the WGM at certain wavelengths when the fiber diameter increases. When the incidence angle is set at 60 deg we observe that the maximum $\mathrm{J}_{\mathrm{sc}}$, shown in Figure $5 \mathrm{a}$, shifts slightly towards larger fiber diameters. However, the general trend is very similar to the one seen when the angle is set to $55 \mathrm{deg}$. Finally, we considered the performance for the FAOC as the separation between adjacent fibers was varied. The $\mathrm{J}_{\mathrm{sc}}$, shown in Figure 6, varies little as the fiber separation is increased up to $300 \mathrm{~nm}$, while it shows a mildly pronounced drop as adjacent fibers get closer to progressively overlap and eventually causing the final FAOC configuration to resemble a planar device. Such drop in $\mathrm{J}_{\mathrm{sc}}$ occurs mostly in between -100 and $-200 \mathrm{~nm}$.

\section{Discussion}

We have demonstrated that an array of fibers can be used as a substrate material to significantly improve light harvesting capacity for

b

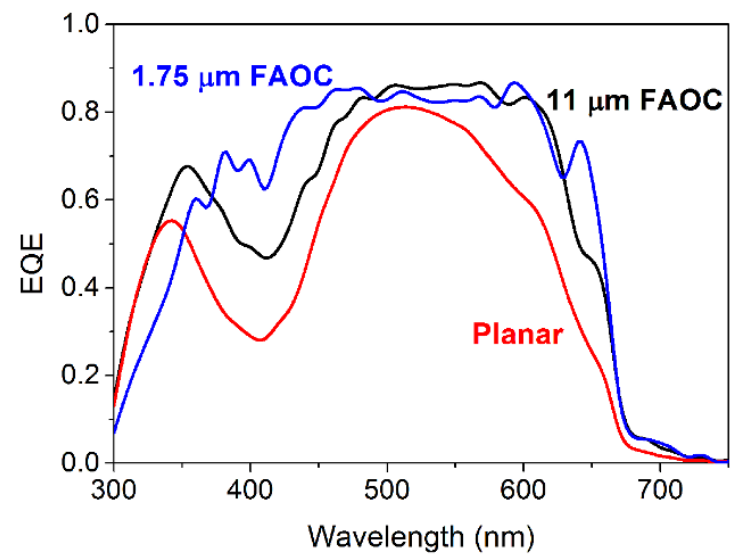

Figure 5 $\mid$ Maximum response of the FAOC as function of fiber diameters. a) Normalized $\mathrm{J}_{\mathrm{sc}}$ at and incidence of 55 deg (circular dots) and 60 deg (square dots) for different fiber diameters of the FAOC. Normalized $\mathrm{J}_{\mathrm{sc}}$ for the planar cell (red dashed line). The green and purple lines are only a guide for the eye. The normalization is to the planar cell at 0 deg incidence. b) EQE at an incidence of 55 deg for the FAOC when the fiber diameter is $1.75 \mu \mathrm{m}$ (blue), when the fiber diameter is $11 \mu \mathrm{m}$ (black), and for the planar cell (red). 


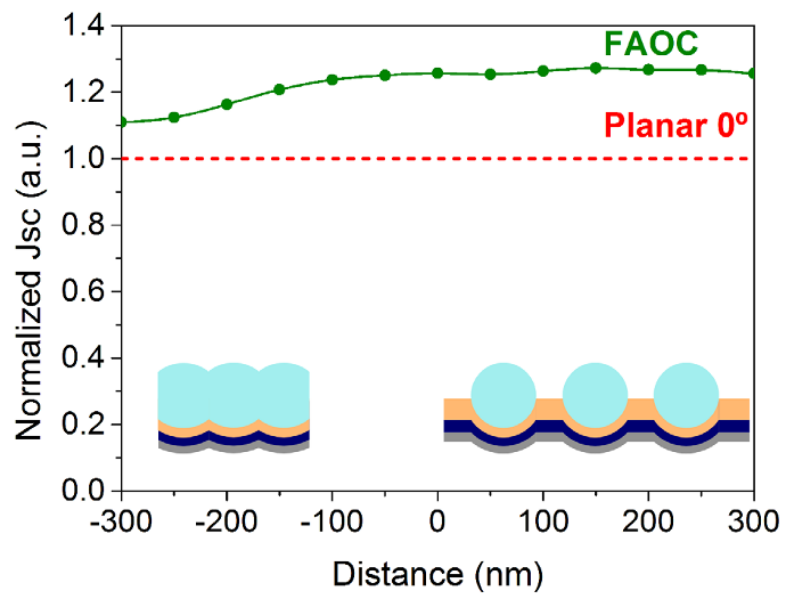

Figure 6 | Electrical response as a function of fiber separation.

Normalized $\mathrm{J}_{\mathrm{sc}}$ at 55 deg incidence for a $1.5 \mu \mathrm{m}$ fiber diameter FAOC (green dots) when the separation between two adjacent fibers is varied. A negative separation corresponds to a fiber overlap (left inset), and a positive separation is for the case when adjacent fibers are connected by a planar cell (right inset). The green line is only a guide for the eye.

organic solar cells when compared to the standard planar device configuration. The incident light is coupled to some low-Q whispering gallery modes of the fibers. Such coupling is effective for a broad range of wavelengths as required when converting light into electricity from a wideband source like the sun. Our numerical study shows that the number of absorbed photons per unit area increases as the sun inclination relative to the cell normal increases from 0 to $55 \mathrm{deg}$. At a Latitude close to 40 deg if one tilts the PV plane by 35 deg relative to the surface of the Earth, the Sunlight will be incident on the PV at approximately 90 deg with respect to the PV plane or, in other words, the Sun would be $55 \mathrm{deg}$ above the horizon line $e^{27}$. This is why at 40 deg latitude the slope of a fixed PV installation on a rooftop or solar filed is, typically, $35 \mathrm{deg}$. On the other hand, the plane of any kind of standard vertical PV installation integrated on a building façade would be $55 \mathrm{deg}$ of such optimal orientation. However, for the FAOC we propose a maximum efficiency would be obtained at $55 \mathrm{deg}$, ensuring an optimal light harvesting for vertical installations. On the other hand, for mechanically fixed photovoltaic (PV) installations the lack of sun tracking leads to a rather inefficient energy harvesting. Indeed, at a Latitude of $40 \mathrm{deg}$ up to $38 \%$ more energy could be harvested by maintaining the plane of the PV installation perpendicular to the sun at all times. In the event that the longitudinal axis of the FAOC would be oriented in the meridian direction, the angular increase in power conversion efficiency would provide, over the course of one day, an energy harvesting capacity $17 \%$ times larger, equivalent to a "half" tracking of the Sun movement. In a future fabrication of the FAOC practical issues such as uniformity, fiber surface roughness, or an increase in the ITO series resistance due to a larger contact area for the FAOC should be taken into account. For instance, to minimize such potential increase in the series resistance of the ITO, a transversal strip of a metal contact across the fibers should be deposited on one end of the FAOC.

\section{Methods}

To study the performance of such FAOC we carried out numerical simulations using the finite element method numerical technique implemented in the commercial software COMSOL Multiphysics ${ }^{\circledR}$. The electromagnetic wave equations were solved in the two-dimensional area corresponding to the transversal section of a single organic cell fiber, shown in Figure $1 \mathrm{~b}$. We assumed the fibers to be made of fused silica and in the numerical modeling the optical parameters for such material were used. The diameter of the fiber was set to $1.5 \mu \mathrm{m}$, except for the cases in which is stated otherwise, while the thicknesses of ITO, P3HT:ICBA, and Al were set each of them to $100 \mathrm{~nm}$, corresponding to the approximate parameters for an optimized performance for the equivalent planar cell ${ }^{28}$. The refractive indices of all materials were taken from our experimental measurements except for the fused silica which was taken from the literature. We imposed periodic conditions (Floquet periodicity) on both lateral sides of the single device to simulate the effect of the array. Such periodic boundary conditions are well justified provided the largest fiber diameter considered in this work is 40 times smaller than the transversal coherence of visible sunlight ${ }^{29}$. Multiple absorbing ports were defined in the upper air boundary for each possible diffraction angle of the periodic structure. This avoided non-physical reflections at the upper boundary coming back to the system fiber plus cell. For the same reason, we kept at least one wavelength distance from the upper boundary to the silica fiber in order to avoid near fields that may not be absorbed by the ports. In-plane (TM) and out-ofplane (TE) polarizations were simulated separately with a power excitation corresponding to one sun. A perfect electric conductor boundary condition was imposed to the lower boundary although its effect is negligible since light is very effectively reflected by the $100 \mathrm{~nm}$ back metal contact. For comparison, a planar OPV was also simulated and the same boundary conditions were applied. In the latter case, light was incident from a fused silica glass substrate and the power of the incident wave was corrected by the corresponding Fresnel coefficient for the air-glass refraction.

1. Nalwa, K. S., Park, J. M., Ho, K. M. \& Chaudhary, S. On Realizing Higher Efficiency Polymer Solar Cells Using a Textured Substrate Platform. Adv. Mater. 23, 112-116 (2011).

2. You, J. et al. Surface Plasmon and Scattering-Enhanced Low-Bandgap Polymer Solar Cell by a Metal Grating Back Electrode. Adv. Energy Mater. 2, 1203-1207 (2012).

3. Gan, Q., Bartoli, F. J. \& Kafafi, Z. H. Plasmonic-Enhanced Organic Photovoltaics: Breaking the 10\% Efficiency Barrier. Adv. Mater. 25, 2385-2396 (2013).

4. Adachi, M. M. et al. Broadband solar absorption enhancement via periodic nanostructuring of electrodes. Sci. Rep. 3, 2928; DOI:10.1038/srep02928 (2013).

5. Lu, L., Luo, Z., Xu, T. \& Yu, L. Cooperative Plasmonic Effect of Ag and Au Nanoparticles on Enhancing Performance of Polymer Solar Cells. Nano Lett. 13, 59-64 (2013).

6. Betancur, R., Romero-Gomez, P., Martinez-Otero, A., Elias, X., Maymó, M. \& Martorell, J. Transparent polymer solar cells employing a layered light-trapping architecture. Nature Photon. 7, 995-1000 (2013).

7. Niggemann, M., Glatthaar, M., Lewer, P., Müller, C., Wagner, J. \& Gombert, A. Functional microprism substrate for organic solar cells. Thin Solid Films. 511512, 628-633 (2009).

8. Grandidier, J., Callahan, D. M., Mundday, J. N. \& Atwater, H. A. Light Absorption Enhancement in Thin-Film Solar Cells Using Whispering Gallery Modes in Dielectric Nanospheres. Adv. Mater. 23, 1272-1276 (2011).

9. Yao, Y. et al. Broadband light management using low-Q whispering gallery modes in spherical nanoshells. Nature Communications. 3, 664 (2012).

10. Spillane, S. M., Kippenberg, T. J. \& Vahala, K. J. Ultralow-threshold Raman laser using a spherical dielectric microcavity. Nature 415, 621-623 (2002).

11. Vahala, K. J. Optical microcavities. Nature 424, 839-846 (2003).

12. Chiasera, A. et al. Spherical whispering-gallery-mode microresonators. Laser Photonics Rev. 4, 457-482 (2010).

13. Wang, Q. J. et al. Whispering-gallery mode resonators for highly unidirectional laser action. PNAS. 107, 22407-22412 (2010).

14. Ta, V. D., Chen, R. \& Sun, H. D. Tuning Whispering Gallery Mode Lasing from Self-Assembled Polymer Droplets. Sci. Rep. 3, 1362 (2013).

15. Ilchenko, V. S., Savchenkov, A. A., Matsko, A. B. \& Maleki, L. Nonlinear Optics and Crystalline Whispering Gallery Mode Cavities. Phys. Rev. Lett. 92, 043903 (2004).

16. Matsko, A. B., Savchenkov, A. A., Strekalov, D., Ilchenko, V. S. \& Maleki, L. Review of Applications of Whispering-Gallery Mode Resonators in Photonics and Nonlinear Optics. IPN Progress Report. 42, 162 (2005).

17. Carmon, T. \& Vahala, K. J. Visible continuous emission from a silica microphotonic device by third-harmonic generation. Nature Physics. 3, 430-435 (2007).

18. Kozyreff, G., Domínguez Juárez, J. L. \& Martorell, J. Whispering-gallery-mode phase matching for surface second-order nonlinear optical processes in spherical microresonators. Phys. Rev. A. 77, 043817 (2008).

19. Kippenberg, T. J., Holzwarth, R. \& Diddams, S. A. Microresonator-Based Optical Frequency Combs. Science, 332, 555 (2011).

20. Domínguez-Juárez, J. L., Kozyreff, G. \& Martorell, J. Whispering gallery microresonators for second harmonic light generation from a low number of small molecules. Nature Commun. 2, 254 (2011).

21. Kozyreff, G., Domínguez-Juárez, J. L. \& Martorell, J. Nonlinear optics in spheres: from second harmonic scattering to quasi-phase matched generation in whispering gallery modes. Laser Photonics Rev. 5, 737-749 (2011).

22. Vollmer, F. \& Arnold, S. Whispering-gallery-mode biosensing: label-free detection down to single molecules. Nature Methods 5, 591-596 (2008).

23. Schliesser, A., Anetsberger, G., Rivière, R., Arcizet, O. \& Kippenberg, T. J. Highsensitivity monitoring of micromechanical vibration using optical whispering gallery mode resonators. New J. Phys. 10, 095015 (2008).

24. Zhu, J.et al. On-chip single nanoparticle detection and sizing by mode splitting in an ultrahigh-Q microresonator. Nature Photon. 4, 46-49 (2009).

25. Lu, T. et al. High sensitivity nanoparticle detection using optical microcavities. PNAS. 108, 5976-5979 (2011).

26. Dantham, V. R., Holler, S., Barbre, C., Keng, D., Kolchenko, V. \& Arnold, S. LabelFree Detection of Single Protein Using a Nanoplasmonic-Photonic Hybrid Microcavity. Nano Lett. 13, 3347-3351 (2013). 
27. Photovoltaic Geographical Information System (EU-JRC PVGIS, Date of access: 06/02/2014) http://re.jrc.ec.europa.eu/pvgis/apps4/pvest.php

28. Betancur, R. et al. Optical interference for the matching of the external and internal quantum efficiencies in organic photovoltaic cells. Sol. Energ. Mat. Sol. C. 104, 87-91 (2012).

29. Mashaal, H., Goldstein, Al., Feuerman, D. \& Gordon, J. M. First direct measurement of the spatial coherence of sunlight. Opt. Lett. 37, 3516-3518 (2012).

\section{Acknowledgments}

This work was supported by the Ministerio de Economía y Competitividad with MAT2011-28665 grant and from the European Comission with Nanophotonics for Energy FP7-248855. Gregory Kozyreff is a Research Associate with the Fonds de la Recherche Scientifique -FNRS (Belgium).

\section{Author contributions}

M.M. and F.J.R. designed the experiment. M.M. carried out the numerical simulations and drew all the figures. P.R.G. and G.K. assisted in data analysis. J.M. and M.M. wrote the manuscript and all authors discussed the results and reviewed the manuscript.

\section{Additional information}

Supplementary information accompanies this paper at http://www.nature.com/ scientificreports

Competing financial interests: The authors declare no competing financial interests.

How to cite this article: Mariano, M., Rodríguez, F.J., Romero-Gomez, P., Kozyreff, G. \& Martorell, J. Light coupling into the Whispering Gallery Modes of a fiber array thin film solar cell for fixed partial Sun tracking. Sci. Rep. 4, 4959; DOI:10.1038/srep04959 (2014).

(c) (1) (2) (2) This work is licensed under a Creative Commons Attribution-NonCommercialShareAlike 3.0 Unported License. The images in this article are included in the article's Creative Commons license, unless indicated otherwise in the image credit; if the image is not included under the Creative Commons license, users will need to obtain permission from the license holder in order to reproduce the image. To view a copy of this license, visit http://creativecommons.org/licenses/by-nc-sa/3.0/ 